\title{
Shape of Abutment Valuation on Designing of Fixed partial Denture: A Study
}

\author{
Dr. Syed Shujaulla ${ }^{1 *}$, Dr. Syeda Tawkhira Tabasum ${ }^{2}$ \\ ${ }^{1}$ Assistant Professor, Department of Prosthetic Dental Sciences, College of Dentistry, Qassim University, Buraidah, Kingdom of saudi arabia \\ ${ }^{2}$ Associate Professor, Department of Periodontology, College of Dentistry, Qassim University, Buraidah, Kingdom of Saudi Arabia
}

DOI: $10.36348 /$ sjodr.2020.v05i07.002

| Received: 23.06.2020 | Accepted: 30.06.2020 | Published: 11.07.2020

*Corresponding author: Dr. Syed Shujaulla

\section{Abstract}

Aim: The target of the present study was to evaluate clinical, radiographic and mounted hypothesis cast assessment of the abutment on pattern of fixed partial denture: objective: Strength is conducted through the abutments to the periodontium. Primary conclude of failures involve poor designing, the consumption of unconventional materials, inadequate tooth preparation, and poor knowledge of biomechanics. Successful survival of abutments for fixed partial dentures needs sensitive diagnostic ability and thorough knowledge of stomatognathic system. Conclusion: Valuation of the abutment choice considered intact part of diagnosis and treatment planning in fixed partial denture

Keywords: Abutment, abutment selection, periodontium, tooth preparation.

Copyright @ 2020: This is an open-access article distributed under the terms of the Creative Commons Attribution license which permits unrestricted use, distribution, and reproduction in any medium for non-commercial use (NonCommercial, or CC-BY-NC) provided the original author and source are credited.

\section{INTRODUCTION}

Substitution of missing teeth symbolizes the need among patients who are seeking for better esthetic and/ or functional teeth. Optional treatments include replacing a single missing tooth; removable partial denture, fixed partial denture or dental implant [9].

Management with removable partial dentures (RPDs) is the most mutual of these choices because it is noninvasive and inexpensive. Nevertheless, treatment with RPDs has a high biological expense with eminent caries incidence and periodontal breakdown of abutment teeth.

The esthetic and functional renewal of a missing tooth or teeth compliments one of the coordination compound for most of the dentists. In few cases, the replacement of the missing tooth or teeth is unmanageable to replace by an implant-supported prosthesis. The possible underlying grounds for this procedure might be due to a local bony defect, an inadequate volume of bone in the edentulous area, occlusal function, systemic disorders, or the socio-economic status or unwillingness of the patient to undergo invasive implant surgery[3].
Fixed prosthodontic treatment esteems an exceptional accomplishment for both, patient and the dentist. The fixed partial denture is one of the most imperative preferred definitive treatment option for a single missing tooth. For many years, FPDs were considered to be the proficient treatment choice for replacing a single missing tooth[1,16].

Survival rate of these FPDs were 75.8 $90.2 \%$ after ten years. The principal reasons for suggesting FPDs are its clinical ease and reduced treatment time and cost. The replacement of the missing teeth in the posterior region is essential as in the anterior segment of the mouth.

It is important to specify the absolute need to fill a space and to perform a costbenefit analysis for any designed restoration not only in commercial damage but also in biological value to tooth structure and the surrounding tissues.

\section{Diagnostic Cast}

Accurate diagnostic casts must be rightly orientated to the transverse hinge axis and plane of occlusion on an articulator to allow eccentric movements exchangeable to those that happen in 
Syed Shujaulla \& Syeda Tawkhira Tabasum; Saudi J Oral Dent Res, July, 2020; 5(7): 310-316

the mouth. This process allows a unsubdivided evaluation of occlusal relationship of dental arches and the abutment teeth.Rotated malposed teeth can be well maintained. The shape and contour of prospective abutment teeth can be envision as well as the alignment and the relationship with the opposing teeth [5].

\section{Roentgenographic Examination}

Periapical and bitewing films are most imperative in choice of abutment teeth. On function additional options, for example TMJ radiographs for patients with TMJ dysfunction and panoramic radiograph can also be conducive.

\section{Intraoral Radiographic Examination Reveals:}

The remaining bone support, root number and morphology (long, short, slender, broad, bifurcated, fused, dilacerated etc.) and root proximity. Quality of supporting bone, trabecular patterns and reactions to functional changes, width of periodontal ligament spaces and evidence of TFO.

Areas of vertical and horizontal osseous resorption and furcation invasions and axial inclination of teeth. (Degree of non parallelism if present), continuity and integrity of lamina dura $[6,7]$.

\section{Factors Influencing Abutment Selection}

The selection and number of abutments are determined by a combination of load-bearing ability of the abutment teeth and the stresses to which they will be subjected. The number of roots, their shape, length, alignment, and bone height has a direct relation to the load- bearing capacity of teeth. The shorter, more tapered the root and lower the bone level, the less satisfactory the tooth will be as an abutment $[7,8]$.

\section{Root Configuration}

Teeth must have adequate occluso-cervical crown length to accomplish sufficient retention. Teeth with short clinical crowns frequently do not render satisfactory retention unless full coverage preparations are used or additional length is achieved through periodontal surgery.

\section{Crown Form}

Some teeth have narrower crown form, which interpose with trailing parallelism, necessitating full coverage retainers to attain their retentive and esthetic qualities. Egs: include anterior teeth with incompletely developed cingula and inadequate proximal walls and mandibular premolars with poorly developed lingual cusps and abrupt proximal surfaces. Besides, few incisors posses very thin highly translucent incisal edges devising use of partial coverage retainers esthetically unacceptable[1,13,15].

\section{Crown- Root Ratio}

This ratio is to evaluate the length of tooth occlusal to the alveolar crest of bone, equivalent to the length of root embedded in bone. As the amount of alveolar bone moves apically, the lever arm of that component part of bone increases, and the probability for injurious lateral forces is increased. The optimum crownroot ratio for tooth to be a fixed partial denture abutment is $2: 3$. A ratio of $1: 1$ is the minimum ratio that is satisfactory for a prospective abutment under normal status. However there are situations where a crown-root ratio of greater than 1:1 might be considered decent.

If the occlusion proposed in FPD is compiled of artificial teeth, occlusal force will be weakened with less stress on the abutment teeth. The occlusal force wield against prosthetic appliance which exhibit to be substantially less than that against natural teeth, For the equal reasons, an abutment tooth with less than desirable crown - root ratio is more likely to be a successful support, FPD if opposing occlusion composed of mobile, periodontally involved teeth than if the opposing teeth are periodontally sound. The crown- root ratio unique is not adequate criteria for measuring a prospective abutment tooth (Penny, Kraal - 1979) The longer the edentulous span and greater the torque on the abutment teeth, the more favorable the crown- root ratio must be. The use of multiple abutments can sometimes compensate for poor crown- root ratio or for long spans $[4,5]$.

\section{Periodontal ligament Surface Area}

This is an imperative point in the assessment of abutment's suitability from a periodontal standpoint. ANTE suggested in 1926 that it was unwise to render a FPD when the root surface area of the abutment was less than the root surface area of the teeth being replaced; this has been adopted and reinforced by other writers (Johnston, Dykema, Shillinburg, Tylman) as ANTE's LAW.

\section{Root Configuration}

Roots that are tolerant labiolinguallly than they are mesiodistally are desirable to roots that are round in cross - section. Multirooted posterior teeth with widely distributed roots will have better periodontal support than roots that are convergent, blunted, or with a conical configuration. A tooth with conical roots can be selected as an abutment if all multiple factors are optimal. A single rooted tooth with irregular configuration or with curvature in the apical third of the root is preferable to the tooth that has almost perfect taper. 
Irregularly shaped, multiple, divergent roots offer better prognosis. A well aligned tooth will provide better support than a tilted one. Alignment can be improved with orthodontic treatment [2,7].

\section{Root Proximities}

There must be decent clearance between the roots of nominated abutments to tolerate the development of physiologic embrasures in final prosthesis. Malpositioned anterior teeth and the mesiobuccal roots of maxillary molars present unfavorable root proximities where desired embrasure form is not possible. Selective extraction or root resection procedures may be the one solution to root proximity.

\section{Periodontal Disease}

After horizontal bone loss due to periodontal disease the PDL supported root surface area is reduced. Because of conical configuration of most roots, when one third of root bone is lost, half the support for the prosthesis is reduced. Furthermore, the forces employed to the supporting area are compromized because of big leverage combined with long clinical crown. Thus potential abutment teeth need careful assessment where substantial bone loss has occurred. In general successful fixed prosthesis can be fabricated on teeth with severely reduced periodontal support, provided the periodontal tissues have returned to good health and long term maintenance has been followed, or it will result in failure. Healthy periodontal tissues are a requirement for all fixed restorations. If the abutment teeth have inadequate bone support, and improper plaque removal by patient will affect the long term prognosis. [11,13].

\section{Periodontal Assessment}

An evaluation of the periodontal tissues should be made. The target is to give a basic screening of the tissues and to receive an indication of the treatment requirements of the patient.

\section{Basic Periodontal Examination}

This is performed clinically using the UNC-15(University of North Carolina) periodontal probe.

\section{Recession}

Any recession or loss of attachment around the teeth should be observed and recorded in the patient's notes.

\section{Long Axis Relationship}

The long axis relationship of abutment teeth should be less than $25^{\circ}-30^{\circ}$ from the parallel. The architecture of periodontal ligament is such that forces are resisted best when they are directed along the long axis of the tooth. A badly inclined tooth will deflect occlusal forces which are injurious. If the force is less and the edentulous span is short, and the tooth is inclined more it can still be used as an abutment. Evaluation of diagnostic casts with a dental surveyor along with the radiographic evaluation.

\section{Mesially Tilted Molars}

Loss of permanent mandibular first molars due to caries early in life is still common. If this space is not replaced, the second molars will tilt mesially. It then becomes challenging to make an acceptable FPD, because the positional relationship no longer allows for parallel path of insertion without interferences from adjacent teeth. The mesial one- half crown preparation, the non - rigid attachment (semi- precision or stress breaker) and the telescopic prosthesis have been suggested in such cases $[1,12,15]$.

The non rigid attachment must not be used indiscriminately. Because of mesial component of force, the female portion of attachment is usually placed on the distal surface of mesial abutment. The cantilever effect on the non- rigid design can tread additional stress on the abutment with the rigid connector, therefore rigid connector is only placed on a strong abutment, and the non rigid design is avoided altogether with long span pontics. Telescopic prosthesis requires radical tooth preparation to allow adequate space for the telescopic coping and the overcasting $[12,16]$.

\section{Clinical Guidelines for Establishing Parallelism}

Developing a common path of insertion on multiple abutments without over reduction of the preparations or exposing the pulp is a fundamental skill that can be mastered. While there is no mystique to the biomechanics of this action, malpositioned, tilted, rotated, and supraerupted teeth increase the demand for planning the path of insertion with a dental surveyor. Once the path of insertion has been established, the base of the cast is scribed with multiple lines parallel to this path to achieve in planning tooth preparations. The following clinical guidelines are suggested:

Select rotary instruments with a standard taper. Diamond stones without a taper must be tilted freehand to void undercut. Freehand tipping avoids the over tapering of the preparations, which cause a loss of retentive form and increase the risk of pulp irritation or exposure.

Occlusal or incisal reduction is accomplished first.They are reduced parallel to the planned occlusal plane. Depth guides are inserted on the buccal and lingual surfaces of all abutment teeth, 
parallel to the planned path of insertion, as indicated by the copied lines on the base of the diagnostic cast.

With the diamond stone held parallel to the planned path of insertion (depth guides), the most unprocurable surface of the most inaccessible abutment is reduced first.

Tooth reduction is done with the diamond stone held parallel to the prepared depth guides; the distal, lingual, facial, and mesial surfaces are reduced systematically. Care should be taken to maintain parallelism between the mesial and distal surfaces of posterior teeth and the facial surfaces of incisors.

After tooth reduction, parallelism is verified with enormous intraoral mirrors. If there is any uncertainty about undercuts, an alginate impression is taken and poured with impression plaster. The plaster cast is assessed on the dental surveyor, and any undercuts are rectified intraorally $[3,5,6]$.

\section{Arch Form}

Restorations involving anterior teeth are figured in the form of an arc. When strength are applied to the pontics, a rotational result occurs on the abutments and a vertical force is exerted on the terminal ends of the fixed partial denture. The equaliser force supplied by the abutments should be the same or may exceed that of the pontics as pointed out by the length of the lever arm.

\section{Span Length}

To avoid or reduce the increased load placed on the periodontal ligament by a long span fixed partial denture, longer spans are usually avoided fixed. Deflection varies directly with the cube of the length and inversely with the cube of the occlusogingival thickness of the pontic. When compared with a fixed partial denture having a single-tooth pontic span, a two-tooth pontic span will deflect 8 times. A three-tooth pontic will bend 27 times as compared to a single tooth pontic.

A pontic with a preconditioned occlusogingival dimension will flex eight times as much if the pontic thickness is halved. A longspan fixed partial denture on short mandibular teeth will have poor clinical outcome. Longer pontic spans produce more torquing forces on the fixed partial denture, especially on the unaccented abutment. To minimize flexing by long and/or thin spans, pontic designs with a greater occlusogingival dimension should be choosen. The prosthesis may also be fabricated of with an alloy with higher yield strength, such as nickelchromium $[3,5,14]$.

All fixed partial dentures, long or short, flex to some point. Because of the forces being applied through the pontics to the abutment teeth, the forces on castings function as retainers for fixed partial dentures are different in magnitude and guidance from those applied to single restorations. The dislodgement of a fixed partial denture retainer tend to move in a mesiodistal direction, as opposed to the more common buccolingual direction of forces on a single restoration. Multiple grooves on the buccal and lingual surfaces are usually marked to overcome this.

Multiple abutments are selected as a means to overcome the disadvantages created by unfavorable crown-root ratio and long span bridges. There are different criteria to be considered if a secondary abutment is used to reenforce the fixed partial denture. A secondary abutment must have at least as much root surface area and as favorable a crown-root ratio as the primary abutment it is intended to re-enforce. The retainers on secondary abutments must be as retentive as the retainers on the primary abutments. When the pontic flexes, tensile forces will be applied to the retainers on the secondary abutments. There should be sufficient crown length and space between adjacent abutments to preclude impingement on the gingiva under the connector.

The arch form also has its effect on the stresses occurring in a fixed partial denture. When pontics lie outside the interabutment axis line, the pontics move as a lever arm, which cause a torque. This is a basic problem in replacing all four maxillary incisors with a fixed partial denture, and it is most articulate in the arch that is pointed in the anterior. This can best be accomplished by gaining additional retention in the opposite direction from the lever arm and at a distance from the interabutment axis equal to the length of the lever arm $[11,15]$

\section{Rigidity}

The lack of sufficient rigidity in a fixed prosthesis is a main cause of failure. Rigidity can be obtained by the selection of proper materials with adequate thickness in order to withstand the occlusal forces acting upon them. A longer metal bar will deform eight times more than the shorter bar when subjected to the same force. If the bar is parallel it is doubled. Rigidity of Fixed partial dentures and conservation of tooth structure may be obtained when the principles are followed 
Margin Location

Sound tooth enamel cannot be amended biologically or esthetically. Consequently when conditions allow, margins of restorations should be placed at the marginal gingiva or a little below it . The near accurate margin for any restorative material may cause inflammation of the gingiva when it is present beneath the free margin.

\section{Occlusal Anatomy}

Occlusal anatomy helps in directing the occlusal forces on the alignment of the teeth. The occlusal surfaces of natural posterior teeth have discrete cusps with many primary and supplemental ridges. The cusps are convex in shape with grooves between the ridges. The natural anatomy should be duplicated in all restorations. The ridges and grooves increase the sharpness and shearing activity of teeth and reduce detrition between opposing surfaces by keeping fatigue contacting area to a minimum. Depicting the natural anatomy permits efficient mastication of food, thus reducing the load transmitted.

\section{Stallard}

Points out that worn-out (attrited) teeth require more muscular power and longer and more masticatory strokes to chew food adequately. Much of this force is directed at right angles to the long axis of the teeth. Properly articulated ridge-bearing cusps will track the food rapidly, with fewer strokes, with much less muscular effort, will direct the occlusal forces in line with the long axis of the teeth. Occlusal forces are related to degree of muscular activity, the patient's habits such as bruxism, the number of teeth being replaced, the leverage on the bridge and adequacy of bone support $[14,15]$.

Excessive occlusal forces cause loosening of prosthesis through flexure or can induce ceramic fracture.The forces can also lead to tooth mobility, particularly in presence of decreased bone support.The buccolingual width of pontics should harmonize with buccolingual dimension of natural unmutilated teeth, and animate the normal buccal and lingual form to the height of contour. Reducing the width of the pontics does not materially reduce the forces transmitted to the abutments, but exerts injurious heavier per unit stress on the restoration and produces conditions improperly contoured teeth[4,6].

\section{Splinting}

Immobilization of teeth by uniting them to one another with soldered retainers to the healthy teeth will reduce and arrest bone loss in compromised teeth. The splinted restoration is hard for patient to clean. Long term presence of permenant splinting may lead to failure of a single abutment or retainer and can jeopardize the entire prosthesis. When in uncertainty do not splint. Hyper mobility is not necessary an indicant for splinting since the mobility can frequently be reduced with an occlusal adjustment. Splinting will not prevent periodontitis and may actually increase the chances of inflammatory disease, since home care will be inhibited. The result is much accelerated by periodontal deterioration of both teeth $[9,16]$.

\section{Pontic Tissue Contact}

The tissue surfaces of the pontic should be convex, smooth and free of porosity. The area of tangency should be minimal, free of pressure and thought of as having saliva touch rather than tissue contact.

\section{Available Tooth Structure}

The size, number and position of carious lesions or restorations in tooth indicates whether full or partial coverage retainers are advised. Extensive defective restorations or fractures require intentional endodontic therapy or post and core fabrication to provide a required retentive and resistant form to the preparations. Crown lengthening maybe indicated to display sound tooth coronal to biologic width when caries, restorations fractures are in propinquity to alveolar crest.

\section{Age of the Patient}

Fixed prosthesis is mostly contraindicated in mouth of adolescents when teeth are not fully erupted or when the pulps are excessively large and prohibit retentive preparations. A space maintainer may be adviced to hold the abutments and opposing teeth in position. However tooth reduction should be kept minimal and prosthesis should be considered temporary because of the large pulp chambers of the developing teeth $[2,3,4,9]$.

\section{Static or Dynamic Occlusion of Teeth}

Abutment teeth are prone to tilting rotation, overeruption, malalignment, attriting and wearing. The patient is asked to close the teeth together and an assessment is made of the occlusal relationship.The occlusion is checked whether it is as canine guided or group function, and any non-working side contacts are observed. The intercuspal location is the relation of the mandible to the maxilla when the teeth are together in maximum intercuspation.

Many patients have a habitual intercuspal relation. Lateral excursions are performed commonly by the canine and the premolar teeth. In canine guidance, the posterior teeth will disocclude in lateral excursions. In group function, a group of teeth, such as the canines 
and premolar teeth; are contacting on the working side (i.e. the side to which the mandible moves). In protrusive movements the anterior teeth move over each other with the posterior teeth disoccluding. However, in tooth wear, the posterior teeth may be in function due to excessive wear of the anterior teeth.

The retruded position of the mandible is where it is in its furthest distal position when contacting the upper teeth. Generally the patient can be led into this position, which is usually 1$2 \mathrm{~mm}$ further posterior to the intercuspal position. Interferences are abnormal contacts that interpose with the smooth movements of the mandible and may be the reason of tooth fracture or cementation failure of a crown etc, or may lead to tooth mobility $[5,7,13]$.

\section{Vitality Testing of the Pulp}

Vitality of the tooth may be tested using either electrical or thermal stimulation.The electric pulp testing is carried on with a machine and the patient becomes part of the circuit when the tip is placed on the tooth. Good electrical contact is achieved by the use of prophylactic paste.

Thermal stimulation may be through either cold or heat, but cold stimulation is advised. This is done using a ice stick or a pledget of cotton wool soaked in ethyl chloride, which will give a quick response.

Nevertheless, a more intense cold stimulus can be provided by use of dry ice. This way the prospective abutment teeth are tested for pulp vitality, if pulp is non-vital it should be endodontically treated before using as abutment for $\operatorname{FPD}[11,13,15]$.

\section{Endodontically Treated Abutments}

Non vital teeth should be endodontically treated before initiating fixed prosthesis. Teeth which require direct pulp capping should also be endodontically treated or it would jeopardize the overall success of treatment.

Such endodontically treated teeth serve well as abutment with post and core foundation for retention and resistance. Failures occur, however particularly on teeth with short roots or small remaining coronal tooth structure. Sometimes its good to remove badly damaged tooth rather than attempting endodontic treatment.

\section{Unrestored Abutments}

An unrestored, caries free tooth is an paragon abutment. It can be prepared conservatively for a potent retentive restoration with optimum esthetics. The edge of retainer can be made without modification to accommodate the existing restorations or caries.

In an adult patient, an unrestored tooth can be safely prepared without jeopardizing the pulp as long as the design and technique of tooth preparation are wisely chosen. Few patients are reluctant to have a perfectly sound tooth cut down to provide anchorage for FPD. In this situation, overall dental health has to be emphasized rather than looking at each tooth individually $[6,8]$.

\section{Abutment Selection for Cantilever FPD}

A cantilever FPD is one that has an abutment or abutments at one end only, with the other end of pontic remaining unattached. This is possibly a destructive design with the lever arm created by pontic and it is frequently misused.

A cantilever pontic can be successfully employed if the primary leverages are understood and provisions are made to control deleterious forces. A classic FPD design is the lateral incisor cantilever pontic supported by a strong canine. A cantilever first premolar pontic can occasionally eliminate the need to organize the canine, thus preserving the natural canine function. In routine three-units FPD, force that is applied to the pontic is distributed equally to the abutment teeth.

If there is only one pontic and it is near the interabutment axis line, less leverage is applied to the abutment teeth or to the retainers than with a cantilever. The pontic acts as a lever to be depressed under forces with a strong occlusal vector.

Prospective abutment teeth for the cantilever FPD must provide average support, tooth preparation must be extremely retentive, occlusal scheme must be close to ideal, the roots should have favorable configuration, long clinical crown, good crown-root ratio and healthy periodontium[7,9,10].

\section{A Cantilever Replacing Maxillary Lateral Incisor}

Canine is used as abutment and can be used as single abutment only if it has good bone support. Should have repose on mesial of pontic against a rest preparation in an inlay or other metallic restoration on the distal of central incisor to prevent rotation of pontic and abutment.

Mesial aspect of pontic is slightly captive around the distal portion of uninvolved central incisor to stabilize pontic faciolingually. Root configuration of central incisor does not make it desirable for cantilever abutment. 


\section{A Cantilever Pontic on First Premolar}

Will work best if occlusal contact is limited to distal fossa. Full veneer retainers are required on both second premolar and molar.

\section{Cantilever FPD Replacing Molar}

It is prepared when no distal molar abutment is available. Most frequently used to replace second molar to prevent supraeruption of opposing teeth. When pontic is located occlusally the adjacent abutment incline to act as fulcrum with tilting tendency on the farthest retainer. To denigrate the leverage effect, the pontic should be kept as small as possible more nearly representing premolar than molar.They should have adequate occlusocervical height to ensure rigid prosthesis. A posterior cantilever pontic places maximum demands on retentive capacity of retainer. Thus its use must be limited to teeth with adequate clinical crown length and abutment for maximum retention.The success of cantilever in the restoration of periodontally weakened dentition is probably due to the fact that these teeth have highly long clinical crowns. While cantilever FPD appear to be conservative, the potential damage to abutment teeth requires that they be used sparingly [11-14].

\section{CONCLUSION}

It can be concluded that the suited survival of abutments influences the prognosis of the treatment. Successful selection of abutments would command and inspire diagnosis and meticulous treatment planning.

\section{REFFERENCE}

1. Al-Quran F. A., Al-Ghalayini R. F., Al-Zubi B.N. (2011). Single-tooth replacement: factors affecting different prosthetic treatment modalities. BMC Oral Health, 11: 34.

2. Fueki K., Yoshida E., Igarashi Y. (2011). A systematic review of prosthetic restoration in patients with shortened dental arches. Jap Dent Sci Rev, 47: 167-174.

3. Singh K., Gupta N., Unnikrishnan, N., Kapoor V., Arora D., Khinnavar P.K. (2014). A conservative treatment approach to replacing a missing anterior tooth. Case Rep dent, 625784.
4. Raza M., Mullah F., Fayyaz M., Akram S. (2015). Complication and their seve rity in patients of conventional metal ceramic fixed dental prosthesis: A clinical study. Pak Oral \& Dental Journal, 35(1): 155-158.

5. Tomruk C.O., Kayahan Z.O., Sencift K. (2014). Patients' knowledge and awareness of dental implants in Ia Turkish subpopulation. J Adv Prosthodont, 6(2): 133-137.

6. Hemmings K., Harrington Z. (2004). Replacement of Imissing teeth with fixed pro theses. Dent Update; 31(3): 137-141.

7. Shillingburg H.T., Sather D.A., Wilson E.L., Cain J.R., Mitchel D.L, Blanco L.J. Kessler J.C.(2012). Fundamental of Fixed Prosthodontics, Ied. I4, Quintessence Publishing Co, Inc.

8. Kalra D., Kalra A., Goel S. (2015). Selecting abutments in fixed prosthodontics - A Review. IJERMDC, 2(1): 21-23.

9. Budtz-Jo"rgensen, E. (1996). Restoration of the partially edentulous mouth-an comparison of overdentures, removable partial dentures, fixed partial dentures and implant treatment. J dent, 24:237-44.

10. WO Stmann B., Budtz-Jørgensen, E., Jepson N., Mushimoto, E., Palmqvist S., Sofou A.(2005). Indications for removable partial dentures: a literature review. Int J Prosthodont, 18:139-45.

11. Budtz-Jørgensen E., Isidor F., Karring T.(1985). Cantilevered fixed partial dentures in a geriatric population: preliminary report. J Prosthet dent, 54:467-73.

12. Himmel R., Pilo R., Assif D., Aviv I. (1992). The cantilever fixed partial denture-a literature review. J Prosthet dent, 67:484-7.

13. Decock V., de Nayer, K., de Boever, J.A., dent, M.(1996). 18-Year longitudinal study of cantilevered fixed restorations. Int $J$ Prosthodont, 9:331-40.

14. Ka"yser A.F.(1981). Shortened dental arches and oral function. J Oral Rehabil 8:457-62.

15. Ka“yser A. F., Battistuzzi P.G., Snoek P.A., Plasmans, P.J. Spanauf, A.J. (1988). The implementation of a problem-oriented treatment plan. Aust Dent J, 33:18-22.

Ka“yser A.F. (1989). The shortened dental arch: a therapeutic concept in reduced dentitions and certain high-risk groups. Int $\mathrm{J}$ Periodontics Restorative dent, 9:427-49... 